\title{
On the Classification of Modulation Schemes using Higher Order Statistics and Support Vector Machines
}

\section{Remziye Büsra CORUK ( $\square$ busra.tezel@atilim.edu.tr )}

Atilim University https://orcid.org/0000-0002-9466-3862

\section{Bengisu Yalcinkaya}

Atilim University: Atilim Universitesi

\section{Mohamed Benzaghta}

Atilim University: Atilim Universitesi

\section{Ali Kara}

Atilim University: Atilim Universitesi

\section{Research Article}

Keywords: Modulation classification, Feature extraction, Support vector machines, Analog modulation, Digital modulation

Posted Date: July 13th, 2021

DOl: https://doi.org/10.21203/rs.3.rs-681327/v1

License: (9) This work is licensed under a Creative Commons Attribution 4.0 International License. Read Full License 


\title{
On the Classification of Modulation Schemes Using Higher Order Statistics and Support Vector Machines
}

\author{
R. B. Coruk - B. Yalcinkaya - M. \\ Benzaghta · A. Kara
}

Received: date / Accepted: date

\begin{abstract}
The recognition of modulation schemes in military and civilian applications is a major task for intelligent receiving systems. Having no previous knowledge of the transmitted signal as well as uncertainties in the channel and the receiver makes the identification of the modulation scheme a difficult task. Various Automatic Modulation Classification (AMC) algorithms have been developed to overcome this challenging task. However, classification with low computational complexity as well as reasonable processing time is still a challenge, especially, for modulation types with similar constellations under realistic channel conditions. In this paper, a feature-based approach along with various classifiers is employed based on statistical features as well as higherorder moments and cumulants. First, some well-known classifiers including Decision Trees (DT), Support Vector Machines (SVM), K-Nearest Neighbors $(\mathrm{KNN})$ and ensemble are evaluated at different SNR values. Then, various forms of SVMs have been utilized. An over-the-air (OTA) recorded dataset consisting of four analog and ten digital modulation schemes are used for test-
\end{abstract}

R. B. Coruk

Department of Electrical and Electronics Engineering, Atilim University, Ankara, Turkey Tel.: +90-312-5868374

E-mail: busra.tezel@atilim.edu.tr

B. Yalcinkaya

Department of Electrical and Electronics Engineering, Atilim University, Ankara, Turkey

Tel.: +90-312-5868374

E-mail: bengisu.yalcinkaya@atilim.edu.tr

M. Benzaghta

Department of Electrical and Electronics Engineering, Atilim University, Ankara, Turkey Tel.: +90-312-5868374

E-mail: mbenzaghta96@gmail.com

A. Kara

Department of Electrical and Electronics Engineering, Gazi University, Ankara, Turkey

Tel.: +90-312-5868374

E-mail: akara@gazi.edu.tr 
ing the proposed method. The classification performance is compared against SNR (0-20 dB). The overall accuracy for quadratic SVM is found to be as high as $98 \%$ at $10 \mathrm{~dB}$. This has been comparable with the reported performance based on simulated dataset. Overall, the comparison of the results with those published in the literature indicates that this is the first paper presenting such a high accuracy with OTA dataset consisting of fourteen modulation schemes.

Keywords Modulation classification - Feature extraction - Support vector machines · Analog modulation · Digital modulation

\section{Introduction}

Automatic Modulation Classification (AMC) plays a vital role in a wide range of applications in military and civil industries. The implementation of AMC can be largely categorized into two groups [1]; likelihood-based (LB) [2] and feature-based (FB) [3] approaches. In the LB approach, hypothesis testing is utilized in conjunction with the likelihood function. Even though this method gives an optimal solution, the probabilistic nature of LB is considered to be computationally heavy. Moreover, it is sensitive to model mismatches such as phase and timing errors along with frequency offsets and noise $[4,5]$. In contrast to the LB approach, computational complexity in the FB approach is much lower, and the implementation is much simpler [6]. The FB method is based on the extraction of features of the received signal, and therefore, this is highly dependent on how much the features are distinctive. Implementation of the FB approach follows feature extraction and classification stages. In the literature, various features have been utilized, for example, statistical features of instantaneous amplitude, phase and frequency characteristics of the received signal including High Order Moments or Cumulants (HOMs/HOCs) or known as Higher-order statistics (HOSs) [7], or spectrum symmetry and [8-10] wavelet transform [11]. The set of features could be basically selected based on modulation type, classification methods, channel effect, etc. [12-21]. Various classifiers including decision tree (DT), k-nearest neighbor (KNN), ensemble classifiers and support vector machines (SVM) have been used in the classification stage of AMC in the literature $[22,36]$. On the other hand, deep learning (DL) has received a great attention in AMC implementations [23,24] while this research focus on ML based classifications only.

When the studies in the literature are examined, mostly they have used the simulated channel effects or Additive White Gaussian Noise (AWGN) channels. However, this does not represent realistic channels. Moreover, modulation schemes studied in the literature were very limited $[5,8,11,16]$, and only HOCs or statistical characteristics were used in feature extraction which may not work for diverse modulation schemes $[6,9,15,19]$. 
In this study, a wide range of over the air (OTA) recorded signals at different Signal to Noise Ratio (SNR) levels are used for modulation classification. A total of 14 modulation schemes consisting of i) analog modulation schemes (Single Sideband, Double Sideband and Frequency Modulation) ii) digital modulation schemes (On-Off Keying, M-ary Amplitude Shift Keying with $\mathrm{M}=4,8$, M-ary Phase Shift Keying with $\mathrm{M}=2,4,32$, M-ary Amplitude Phase Shift Keying with $M=16,32$, Offset Quadrature Phase Shift Keying and Gaussian Minimum Shift Keying) have been used. The dataset collected as OTA under real propagation effects is available [25]. Statistical features including mean, variance, skewness and kurtosis as well as moments and cumulants up to 8th order are employed. 17 classifiers including the derivatives of DT, KNN, SVM and ensemble classifiers have been employed and their performance has been compared at three different SNR levels $(0,10$ and 20 $\mathrm{dB}$ ). Linear, quadratic and cubic SVMs are used for further processing due to their higher classification accuracy. The effects of the feature sets on the classification of particular modulation types are evaluated as well. Finally, the performance of the classification has been compared with the published works in the literature.

The rest of the paper is organized as follows: In Section II, the proposed method including feature extraction and classification is given. Section III present the classification performance and discusses the results in comparison with the literature. Section IV draws some conclusions along with future work.

\section{Proposed Method}

\subsection{Signal Preprocessing}

Dataset contains over-the-air (OTA) recorded signals in IQ (In-phase and Quadrature) form [25] . First of all, the time domain complex signal can be formed as $[12,13]$

$$
s(n)=I(n)+j Q(n)
$$

where $I(n)$ and $Q(n)$ are the in phase $(I)$ and the quadrature $(Q)$ components of the samples of the signal, and $n$ is the sample (time) index. Instantaneous amplitude $a(n)$, phase $\phi(n)$ and frequency $f(n)$ characteristics of the complex signal can be derived as follows

$$
\begin{gathered}
a(n)=\sqrt{I^{2}(n)+Q^{2}(n)} \\
\phi(n)=\tan ^{-1}\left[\frac{Q(n)}{I(n)}\right] \\
f(n)=\frac{1}{2 \pi} \frac{\phi(n)-\phi(n-1)}{\Delta n}
\end{gathered}
$$


In the next step, these must be normalized by subtracting their mean values in order to avoid biases superimposed in the data collection stage. Following the method in [26], the normalized instantaneous signal characteristics can be written as

$$
\begin{gathered}
a_{c}(n)=a(n)-\mu_{a} \\
f_{c}(n)=f(n)-\mu_{f} \\
\phi_{n l}(n)=\phi(n)-2 \pi \mu_{f}(n) \Delta_{t} \\
\phi_{c n l}(n)=\phi_{n l}(n)-\mu_{\phi_{n l}}
\end{gathered}
$$

\subsection{Feature Extraction and Classification}

Derivation of statistical features instead of the complex signal characteristics derived previously provides a reduction in the computational complexity as well as feature space dimension. Therefore, the following features, namely, mean $\left(\mu_{x}\right)$, variance $\left(\sigma_{x}^{2}\right)$, skewness $\left(\gamma_{x}\right)$, and kurtosis $\left(\kappa_{x}\right)$, respectively, are derived for any signal characteristic, $x(k)$.

$$
\begin{gathered}
\mu_{x}=\frac{1}{N_{x}} \sum_{k=1}^{N_{x}} x(k) \\
\sigma_{x}^{2}=\frac{1}{N_{x}} \sum_{k=1}^{N_{x}}[x(k)-\bar{x}]^{2} \\
\gamma_{x}=\frac{1}{\sigma_{x}^{3} N_{x}} \sum_{k=1}^{N_{x}}[x(k)-\bar{x}]^{3} \\
\kappa_{x}=\frac{1}{\sigma_{x}^{4} N_{x}} \sum_{k=1}^{N_{x}}[x(k)-\bar{x}]^{4}
\end{gathered}
$$

where $\bar{x}$ is the mean of $x(k)[26]$.

Additionally, higher-order moments (HOMs) and cumulants (HOCs) are included to achieve better performance in classification, especially, for high-order modulation schemes. HOMs can be written as

$$
M_{p q}=E\left[s^{p-q}\left(s^{*}\right)^{q}\right]
$$

where $E[]$ denotes expectation, $*$ denotes complex conjugate, and $p, q$ determine the order of the moment. The HOMs play a role in obtaining HOCs [12] as well. For example, the following defines one of the HOCs $\left(C_{42}\right)$

$$
C_{42}=M_{42}-\left|M_{20}\right|-2 M_{21}^{2}
$$


Table 1 Features

\begin{tabular}{lll}
\hline Statistical & Moments & Cumulants \\
\hline$\mu_{x}$ & $M_{20}, M_{21}$ & $C_{20}, C_{21}$ \\
$\sigma_{x}^{2}$ & $M_{40}, M_{41}, M_{42}, M_{43}$ & $C_{40}, C_{41}, C_{42}$ \\
$\gamma_{x}$ & $M_{60}, M_{61}, M_{62}, M_{63}$ & $C_{60}, C_{61}, C_{62}, C_{63}$ \\
$\kappa_{x}$ & $M_{80}, M_{81}, M_{82}, M_{83}, M_{84}$ & $C_{80}, C_{81}, C_{82}, C_{83}, C_{84}$ \\
\hline
\end{tabular}

Table 2 Properties of multiclass SVM classifiers

\begin{tabular}{lllll}
\hline Classifier Type & Prediction Speed & Memory Usage & Interpretability & Model Flexibility \\
\hline Linear SVM & Medium & Medium & Easy & Low \\
Quadratic SVM & Slow & Large & Hard & Medium \\
Cubic SVM & Slow & Large & Hard & Medium \\
\hline
\end{tabular}

Based on [27], the amplitudes of the HOMs and HOCs are taken into account. Then, all features are listed in Table I.

In the next step, Support Vector Machines (SVMs) are used in classification. SVM is known as a supervised machine learning algorithm, and it show high performance when classifying the noisy and high dimensional data samples. It uses hyperplanes to separates data among the classes. The SVM logic is that all modulation classes are mapped with kernel function called transformations with which the separation of classes is conducted. If the linear kernel is used, SVM is called linear. If the non-linear kernel is used, SVM is called non-linear (i.e., polynomial and Gaussian type kernels). In linear SVM classifier, the linear kernel can be defined by

$$
F(a, w)=a^{T} w
$$

where $a=\left[a_{1}, a_{2}, \ldots a_{k}\right]$ is input feature vector and $w$ is the weight vector. The weight vector is optimized through training by designing the hyperplane to attain maximum separation. This optimization reduces computational complexity of the training. The kernel concept helps reduce computational complexity caused by nonlinear SVM in converting the dataset to a high-dimensional space. Polynomial kernel functions used for nonlinear SVM classifiers can be given in the following form

$$
K(x, y)=\left(\gamma X^{T} Y+r\right)^{d}, \gamma>0
$$

where $d$ is the degree of the polynomial. Then, the degree of the polynomial is 3 and 4 for cubic SVM and quadratic SVM, respectively [36]. Basically, SVM uses a set of binary classification sub-problems. A comparison regarding with multi-class SVM is listed in Table II. 


\section{Results and Discussion}

Based on the features and classifiers described in the previous section, overthe-air modulated signals of 14 modulation types are classified. Each modulated signal record contains 1024 IQ samples, and there are 4096 records for each modulated signal at each SNR value. One feature is extracted from each record. The records of each modulated signal are collected within 0-20 dB SNR range with an increment of $2 \mathrm{~dB}$. In this way, the performance of the classification system can be evaluated with respect to SNR variation.

Firstly, 17 classifiers including DT, SVM, KNN and ensemble are evaluated at different SNR values as shown in Table III. It can be seen that the polynomial derivations of SVM is outperform all others. The memory requirement of DT is considered to be small while its prediction speed is fast enough. The depth of the tree can be increased to obtain higher performance but this could lead to overfitting. Based on the number of leaves, DTs can be named as coarse, medium and fine. It seems that the performance of the fine tree is better than the coarse and medium tree for relatively large number of classes. KNN classifiers exploit the distance of neighboring classified samples. The performance of these classifiers are dependent on the number of neighbors, the function of distance weighting and distance metric. Generally, lower the dataset dimension higher the performance is. Their memory requirement and complexity is high and prediction speed is slow compared with DT classifiers. On the other hand, ensemble classifiers can be considered as a combination of multiple learning algorithms to achieve better better prediction performance. Their interpretability is hard while the prediction speed and memory requirement are highly dependent on the algorithms. Two commonly used algorithms are most popular; Boosted and Bagged trees. In Boosted trees, each model is based on incrementally built ensemble which is created by training previously misclassified models. On the other hand, in Bagged Trees, each learner is trained in parallel with the others by a randomly selected subset. Bagged trees use random forest algorithm while Boosted trees use discriminant learners. With lower memory requirements, two more classifiers, namely, Subspace Discriminant and RUSBoosted Trees are widely used in image processing. The prediction speed of the RUSBoosted trees are faster. Moreover, their flexibility as well as the accuracy could be increased by increasing the number of learners. Highly flexible algorithms can model small changes caused by noise which is major limitation in modulation classification. This leads to overfitting problem while classifying noisy signals. For this reason, 5-fold cross-validation is used to resolve this problem. Among these classifiers, it has been observed that Fine Gaussian SVM, Fine KNN, Weighted KNN, Bagged Trees, and Cubic SVM classifiers are tend to be overfitting. Having higher accuracy at low and high SNR ranges, Linear, Quadratic and Cubic SVM classifiers are found to be outperform all others.

The performance of the three classifiers (linear, quadratic and cubic) is shown in Fig. 1 within the SNR range of 0-20 dB. They show similar performance at low SNR values (up to $8 \mathrm{~dB}$ ), and then the linear SVM shows slightly 
Table 3 Performance comparison of classifiers at different SNR values

\begin{tabular}{llll}
\hline \multirow{2}{*}{ Classifier Type } & \multicolumn{3}{c}{ Performances $(\%)$} \\
\cline { 2 - 4 } & $0 \mathrm{~dB}$ & $10 \mathrm{~dB}$ & $20 \mathrm{~dB}$ \\
\hline Fine Tree & 57.5 & 92.6 & 93.6 \\
Medium Tree & 53.2 & 89.0 & 90.6 \\
Linear SVM & 60.5 & 94.3 & 96.0 \\
Quadratic SVM & 61.8 & 96.1 & 96.7 \\
Cubic SVM & 58.9 & 95.8 & 96.5 \\
Fine Gaussian SVM & 45.9 & 87.8 & 88.2 \\
Medium Gaussian SVM & 61.9 & 94.5 & 95.6 \\
Coarse Gaussian SVM & 61.3 & 91.8 & 94.6 \\
Fine KNN & 47.4 & 83.0 & 88.0 \\
Medium KNN & 54.2 & 85.1 & 89.5 \\
Coarse KNN & 56.5 & 83.7 & 89.1 \\
Cosine KNN & 53.0 & 85.2 & 90 \\
Weighted KNN & 54.4 & 85.6 & 90.1 \\
Boosted Trees & 56.1 & 91.5 & 92.7 \\
Baagged Trees & 59.1 & 94.1 & 95.0 \\
Subspace Discriminant & 57.7 & 82.5 & 88.9 \\
RUSBoosted Trees & 53.2 & 89.0 & 90.6 \\
\hline
\end{tabular}

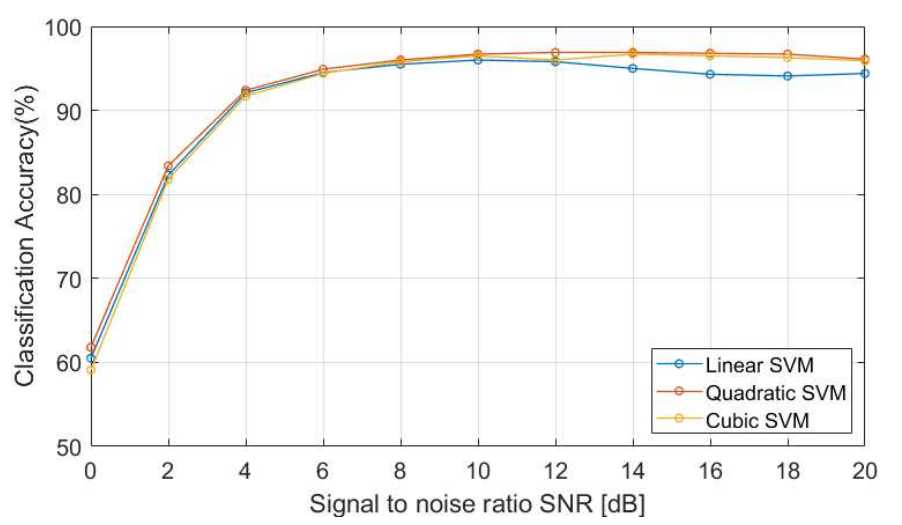

Fig. 1 Classification performances of different SVM classifiers at 0-20 dB

lower performance. Overall, all classifiers achieve around 95\% accuracy above $6 \mathrm{~dB}$ SNR. It seems that the lower bound of the classifiers would be somewhere around $2 \mathrm{~dB}$ where the accuracy drops to $83 \%$. In this evaluation study, $80 \%$ of the records are used to train the network while the remaining (20\%) is used for testing.

Next, it could be interesting to see how training data size could affect the performance of the classification. It is known that the size of the training data could improve the classification accuracy to some extent. For this purpose, based on previous analysis of SVM, the quadratic SVM was picked up and was trained with different size of the training dataset. There is no major difference between the classification accuracy for $20 \%$ training data (819 out of 4096 records) and $60 \%$ training data (2458 out of 4096 records) as shown in 


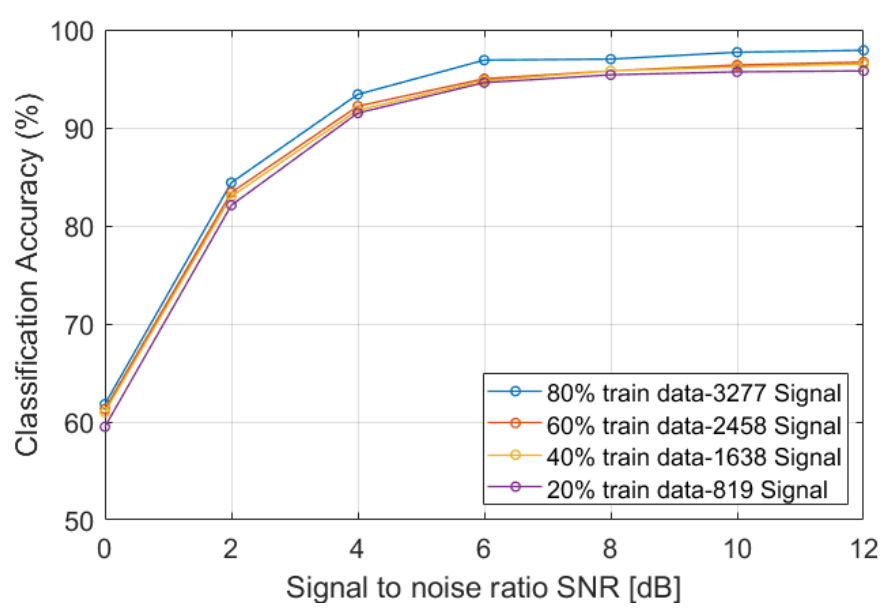

Fig. 2 Classification performances of Quadratic SVM for different training data sizes

Fig.2. However, the classification accuracy increases slightly (2-3\%) when the training data is $80 \%$ of the total data (3277 out of 4096 records). Here, 5 -fold cross-validation was also applied to avoid overfitting. The processing time took around 3 hours which is a very adequate value.

Shown in Fig.3 and Fig.4 are confusion matrices of the quadratic SVM classifier at $0 \mathrm{~dB}$ and $10 \mathrm{~dB}$ SNR, respectively. At low SNR (0 dB), some of the modulation schemes including 32PSK, 16APSK, GMSK, OQPSK as well as DSB analog amplitude modulation schemes have very low classification accuracy (between $30 \%-60 \%$ and as low as $28 \%$ for a few other schemes). The classification accuracy increases significantly ( $87.5 \%$ or above) when the SNR increases to $10 \mathrm{~dB}$ (Fig.4). FM and GMSK have 100\% classification accuracy at $10 \mathrm{~dB}$ SNR. Furthermore, OOK, BPSK, QPSK, AM-SSB-SC and OQPSK have similar classification accuracy $(99 \%)$. When both confusion matrices are examined, it can be seen that the classification accuracy of OOK and AMSSB-SC is almost independent of SNR variations. This could be attributed to the constellation diagrams of the modulation schemes. Overall, average classification accuracy increases to $98 \%$ from $61.8 \%$ when the SNR increases from $0 \mathrm{~dB}$ to $10 \mathrm{~dB}$.

The classification performance is highly dependent on the features. For this purpose, the effects of various feature sets on the classification performance are examined. Mean, skewness, variance, kurtosis constitute the statistical features set. On the other hand, the effects of higher-order moments and cumulants set need to be studied carefully. At low SNR, the use of the statistical features set without moments and cumulants may not work well for OOK, 4ASK, 8ASK, and QPSK modulations. The use of moments and/or cumulants greatly increase the classification performance. For BPSK, 16APSK, and OQPSK, it is found that cumulants perform poorly whereas the use of moments in both low and high SNR significantly increases the classification 


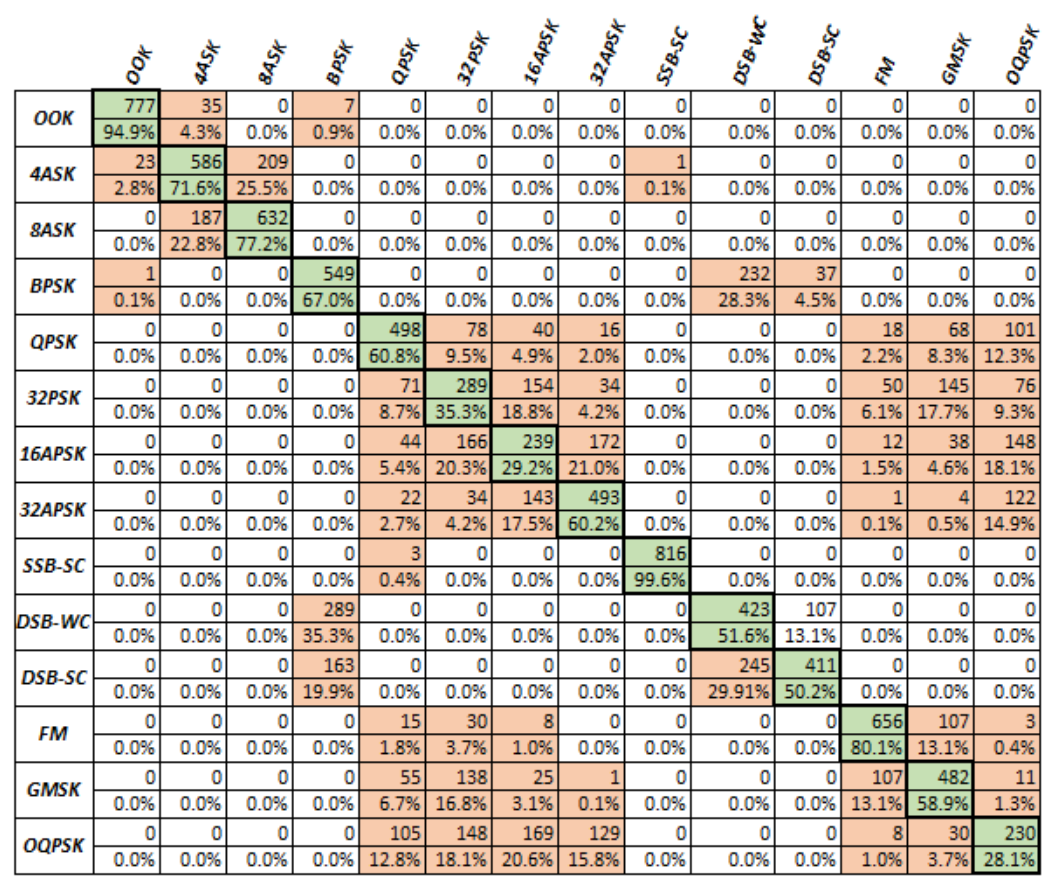

Fig. 3 Confusion matrix at $0 \mathrm{~dB}$

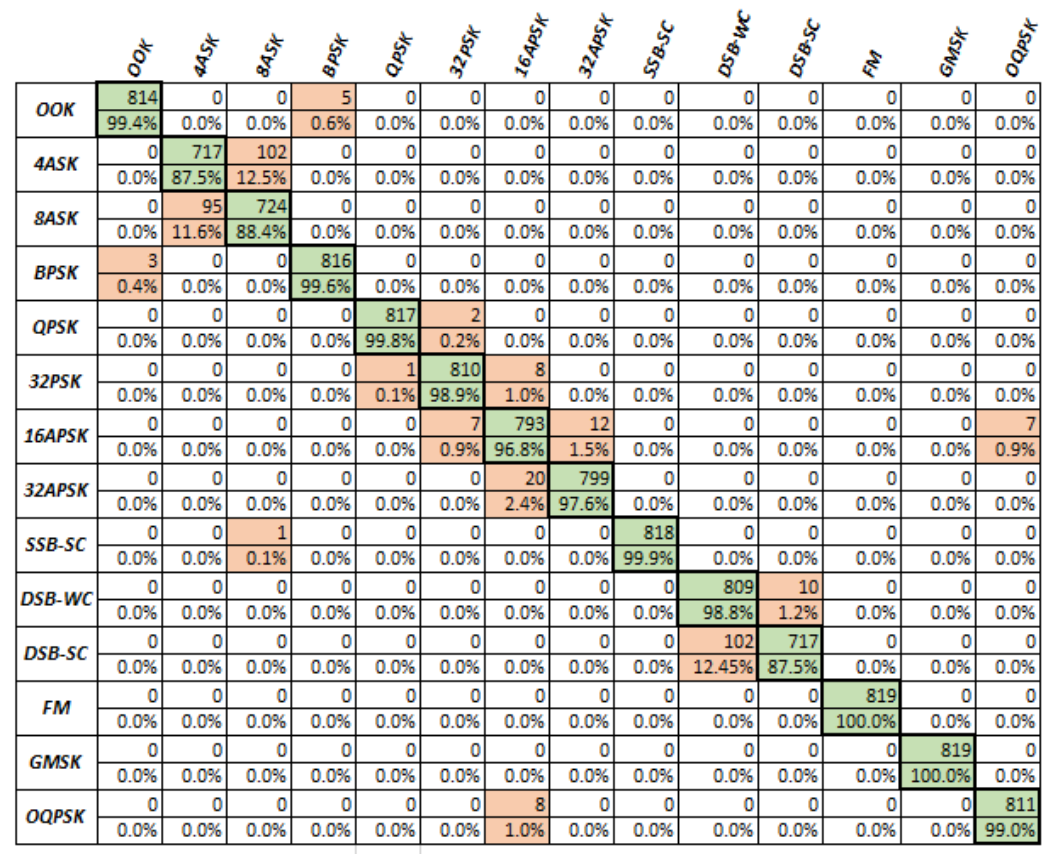

Fig. 4 Confusion matrix at $10 \mathrm{~dB}$ 

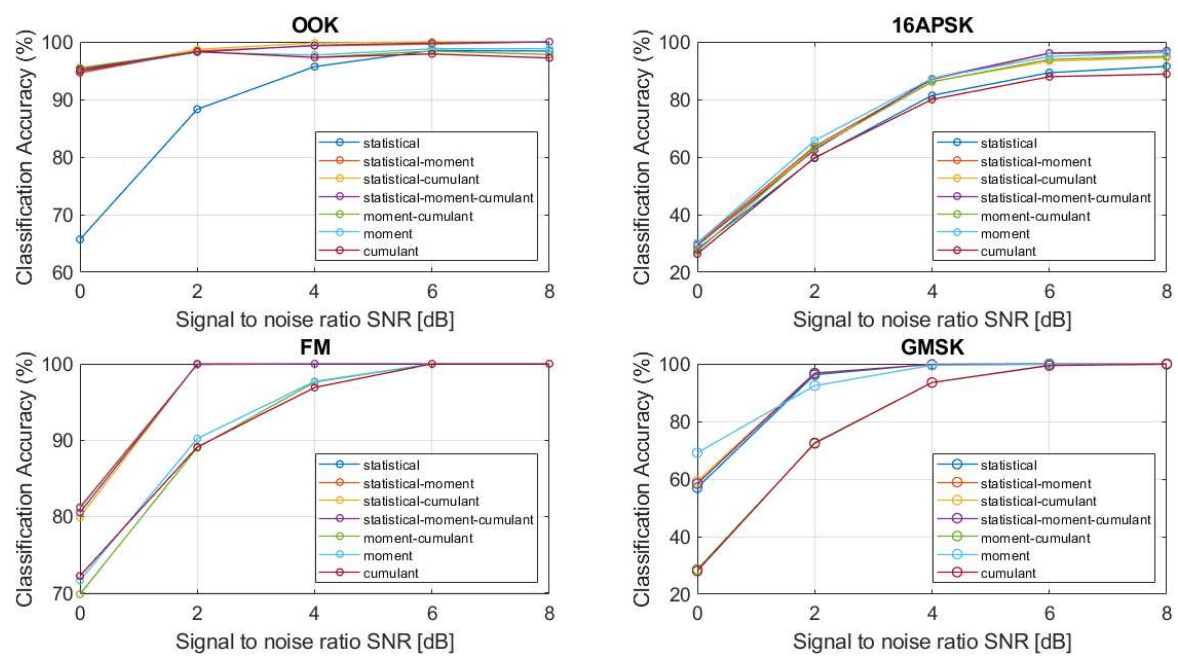

Fig. 5 Feature assessments for four modulation types

performance. For 32PSK, 32APSK, FM, SSBSC modulations, the use of statistical features along with moments and cumulants gives sufficient classification performance. For GMSK modulation, the use of cumulants alone was insufficient in achieving high accuracy while moments or statistical features alone are sufficient in achieving high classification performance. It should be noted that the classification accuracy at SNR values above $6 \mathrm{~dB}$ is almost independent of feature sets for DSBSC, DSBWC, SSBSC, BPSK, 32PSK, 32APSK and OOK. Shown in Fig.5 presents how different feature sets perform for OOK, 16APSK, FM, and GMSK modulation schemes to set an example.

Finally, performances of the modulation classification methods, including ML and DL, of published works in the literature are summarized in Table IV. In order to demonstrate the contribution of this study, the table needs to be examined carefully. It can be concluded that

- Some of the works consider either only low order modulation schemes [14, $29,35]$ and/or limited number of modulation schemes [15, 16, 30, 31, 32, $36,37]$.

- Many [15, 16, 24, 30, 31, 32, 33, 34, 35, 36, 37] use simulated channel effects with computer generated data rather than OTA.

- SVM based classifications [16, 34, 32, 35] generally achieve 90-95\% accuracy but only for limited number of modulation classes, and/or with synthetically simulated channel.

- One [34] presents 90\% classification accuracy at the same SNR level, with similar set of modulation classes, but only under simulated channel effects.

- One another [35] presents 97\% classification accuracy but considers only ASK and PSK modulation schemes. 
Table 4 Comparison of Classification Performances

\begin{tabular}{|c|c|c|c|c|}
\hline Ref. & Dataset & Method & Acc. & SNR \\
\hline 14 & $\begin{array}{l}\text { BPSK, QPSK, OQPSK, MSK, } \pi / 4- \\
\text { QPSK, 8-PSK, 16-QAM (computer } \\
\text { generated Rayleigh fading channel) }\end{array}$ & $\begin{array}{l}\text { Tree-Based Classifier - } \\
\text { Cyclic cumulant }\end{array}$ & $\sim 90 \%$ & $10 \mathrm{~dB}$ \\
\hline 15 & $\begin{array}{l}\text { BPSK, QPSK, 16-QAM, 64-QAM } \\
\text { (computer generated Rayleigh fading } \\
\text { channel) }\end{array}$ & $\begin{array}{l}\text { Softmax Classifier - } \\
\text { Stacked convolutional } \\
\text { auto-encoders } \\
\text { (SCAEs) and } \\
\text { sixth-order cumulants }\end{array}$ & $\sim 90 \%$ & $10 \mathrm{~dB}$ \\
\hline 16 & $\begin{array}{l}\text { BPSK, QPSK, 8PSK, 16QAM, } \\
\text { 64QAM (computer generated fading } \\
\text { channel) }\end{array}$ & $\begin{array}{l}\text { SVM Classifier- C40, } \\
\text { C41, C42, C63 features }\end{array}$ & $\sim 56 \%$ & $5 \mathrm{~dB}$ \\
\hline 24 & $\begin{array}{l}\text { BPSK, QPSK, 8PSK, AM-DSB, } \\
\text { CPFSK, GFSK, PAM4, QAM16, } \\
\text { QAM64, WBFM (computer generated } \\
\text { multipath fading channel) }\end{array}$ & $\mathrm{CNN}$ & $\sim 82 \%$ & $10 \mathrm{~dB}$ \\
\hline 29 & $\begin{array}{l}\text { CW, AM, FM, SSB, FSK2, FSK } 4 \text {, } \\
\text { PSK2, PSK4, OOK, QAM16, QAM } 32 \\
\text { (computer generated) }\end{array}$ & $\begin{array}{l}\text { Hierarchical NN - } \\
\text { Instantaneous and } \\
\text { demodulation domain } \\
\text { features }\end{array}$ & $\sim 93 \%$ & $7 \mathrm{~dB}$ \\
\hline 29 & $\begin{array}{l}\text { AM, CW, FM, FSK2, OOK, PSK2 } \\
\text { (Real propagation effect) }\end{array}$ & $\begin{array}{c}\text { Hierarchical NN - } \\
\text { Instantaneous and } \\
\text { demodulation domain } \\
\text { features }\end{array}$ & $\sim 97 \%$ & $7 \mathrm{~dB}$ \\
\hline 30 & $\begin{array}{l}\text { BPSK, QPSK, 8PSK, 4QAM, 16QAM, } \\
64 Q A M \text { (computer generated fading } \\
\text { channel) }\end{array}$ & NPLF Classifier & $\sim 93 \%$ & $10 \mathrm{~dB}$ \\
\hline 31 & $\begin{array}{l}\text { BPSK, QPSK, 16QAM, 64QAM (com- } \\
\text { puter generated AWGN) }\end{array}$ & GPKNN-Cumulants & $98 \%$ & $10 \mathrm{~dB}$ \\
\hline 31 & $\begin{array}{l}\text { BPSK, QPSK, 16QAM, 64QAM (com- } \\
\text { puter generated AWGN) }\end{array}$ & $\mathrm{KNN}$ & $93 \%$ & $10 \mathrm{~dB}$ \\
\hline 32 & $\begin{array}{l}\text { BPSK, QPSK, 16QAM, 64QAM (com- } \\
\text { puter generated AWGN) }\end{array}$ & Naive Bayes & $94.4 \%$ & $10 \mathrm{~dB}$ \\
\hline 32 & $\begin{array}{l}\text { BPSK, QPSK, 16QAM, 64QAM (com- } \\
\text { puter generated AWGN) }\end{array}$ & SVM Classifier & $94.8 \%$ & $10 \mathrm{~dB}$ \\
\hline 33 & $\begin{array}{l}\text { 2FSK, 4FSK, 8FSK, OQPSK, 2PSK, } \\
\text { 4PSK, 8PSK, 16APSK, 32APSK, } \\
\text { 16QAM,32QAM, 64QAM (computer } \\
\text { generated HF noise) }\end{array}$ & $\begin{array}{l}\text { Decision Tree- } \\
\text { Constellation based } \\
\text { features }\end{array}$ & $\sim 89 \%$ & $10 \mathrm{~dB}$ \\
\hline 34 & $\begin{array}{l}\text { BPSK, QPSK, 8PSK, AM-SSB, AM- } \\
\text { DSB, BFSK, CPFSK, GFSK, PAM4, } \\
\text { QAM16, QAM64, WBFM (computer } \\
\text { generated multipath fading channel) }\end{array}$ & $\mathrm{DNN}$ & $87.4 \%$ & $10 \mathrm{~dB}$ \\
\hline 34 & $\begin{array}{l}\text { BPSK, QPSK, 8PSK, AM-SSB, AM- } \\
\text { DSB, BFSK, CPFSK, GFSK, PAM4, } \\
\text { QAM16, QAM64, WBFM (computer } \\
\text { generated multipath fading channel) }\end{array}$ & Expert-SVM & $90 \%$ & $10 \mathrm{~dB}$ \\
\hline 34 & $\begin{array}{l}\text { BPSK, QPSK, 8PSK, AM-SSB, AM- } \\
\text { DSB, BFSK, CPFSK, GFSK, PAM4, } \\
\text { QAM16, QAM64, WBFM (computer } \\
\text { generated multipath fading channel) }\end{array}$ & Expert-Naive Bayes & $\sim 60 \%$ & $10 \mathrm{~dB}$ \\
\hline 35 & $\begin{array}{l}\text { M-ASK, M-FSK, M-PSK }(\mathrm{M}=2 \text {, } \\
4,8), 16-\mathrm{QAM} \text { (computer generated } \\
\text { AWGN) }\end{array}$ & SVM classifier & $97 \%$ & $10 \mathrm{~dB}$ \\
\hline 36 & $\begin{array}{l}\text { BPSK, QPSK, 8PSK, 4QAM, 16QAM, } \\
64 \mathrm{QAM} \text { (computer generated multi } \\
\text { fading) }\end{array}$ & $\begin{array}{l}\text { SVM classifier - } \\
\text { moment and cumulant }\end{array}$ & $99.4 \%$ & $10 \mathrm{~dB}$ \\
\hline 37 & $\begin{array}{l}\text { MFSK } \quad(M=2,4,8) \quad \text { (computer gener- } \\
\text { ated) }\end{array}$ & $\begin{array}{c}\text { K-means Clustering - } \\
\text { Discrete Wavelet } \\
\text { Transform (DWT) } \\
\text { based Feature }\end{array}$ & $100 \%$ & $10 \mathrm{~dB}$ \\
\hline $\begin{array}{l}\text { This } \\
\text { Work }\end{array}$ & $\begin{array}{l}\text { OOK, 4ASK, 8ASK, BPSK, QPSK, } \\
\text { 32-PSK, 16-APSK, 32-APSK, AM- } \\
\text { SSB-SC, AM-DSB-WC, AM-DSB-SC, } \\
\text { FM, GMSK, OQPSK (Real propaga- } \\
\text { tion effect) }\end{array}$ & $\begin{array}{l}\text { Higher-order } \\
\text { Statistics(HOSs) } \\
\text { features - SVM } \\
\text { classifier }\end{array}$ & $98 \%$ & $10 \mathrm{~dB}$ \\
\hline
\end{tabular}




\section{Conclusions}

This study presents the classification results of 14 modulation schemes based on an over the air dataset (OTA). The assessment of various feature sets including higher-order statistics, moments and cumulants is presented as well. The performances of three SVMs are examined for the classification of ana$\log$ and digital modulation schemes. The performance of the proposed work is compared with the published works in the literature from different aspects including the number of modulation schemes, diversity of modulation schemes, channel models, SNR levels and classifier types as well as accuracy. The classification performance of the prosed study outperforms all reported works with more realistic channel effects and an extended number of modulation schemes. Overall, the results show that modulation classification based on robust features including statistical features and HOMs/HOCs along with SVM has the potential to play an important role in many automatic modulation classification (AMC) applications in realistic radio channels. On the other hand, selective features may reduce computational complexity and processing time for some applications.

\section{Conflict of interest}

The authors declare that they have no conflict of interest.

\section{References}

1. Dobre, O. A., Abdi, A., Bar-Ness, Y., \& Su, W. (2007). Survey of automatic modulation classification techniques: classical approaches and new trends. IET communications, 1(2), $137-156$.

2. Xu, J. L., Su, W., \& Zhou, M. (2010). Likelihood-ratio approaches to automatic modulation classification. IEEE Transactions on Systems, Man, and Cybernetics, Part C (Applications and Reviews), 41(4), 455-469.

3. Hazza, A., Shoaib, M., Alshebeili, S. A., \& Fahad, A. (2013, February). An overview of feature-based methods for digital modulation classification. In 2013 1st international conference on communications, signal processing, and their applications (ICCSPA) (pp. 1-6). IEEE.

4. Zhang, J., Wang, F., Zhong, Z., \& Wang, S. (2018). Continuous phase modulation classification via baum-welch algorithm. IEEE Communications Letters, 22(7), 1390-1393.

5. Kim, S. J., \& Yoon, D. (2016, October). Automatic modulation classification in practical wireless channels. In 2016 International Conference on Information and Communication Technology Convergence (ICTC) (pp. 915-917). IEEE.

6. Abdelmutalab, A., Assaleh, K., \& El-Tarhuni, M. (2016). Automatic modulation classification based on high order cumulants and hierarchical polynomial classifiers. Physical Communication, 21, 10-18.

7. Tezel, R.B. (2020) . Performance Analysis of Higher-Order Statistical Features in Classification of Some Modulation Type (Master's thesis, Atilim University) doi: 10.13140/RG.2.2.19523.32803

8. Jiang, W. H., Tong, F., Dong, Y. Z., \& Zhang, G. Q. (2018). Modulation recognition of non-cooperation underwater acoustic communication signals using principal component analysis. Applied Acoustics, 138, 209-215. 
9. Kubankova, A., Kubanek, D., \& Prinosil, J. (2011, August). Digital modulation classification based on characteristic features and GentleBoost algorithm. In 2011 34th International Conference on Telecommunications and Signal Processing (TSP) (pp. 448-451). IEEE.

10. Nandi, A. K., \& Azzouz, E. E. (1998). Algorithms for automatic modulation recognition of communication signals. IEEE Transactions on communications, 46(4), 431-436.

11. Fucai, Z., Yihua, H., \& Shiqi, H. (2008). Classification using wavelet packet decomposition and support vector machine for digital modulations. Journal of Systems Engineering and Electronics, 19(5), 914-918.

12. Ali, A. M., Uzundurukan, E., \& Kara, A. (2019). Assessment of features and classifiers for Bluetooth RF fingerprinting. IEEE Access, 7, 50524-50535.

13. Aghnaiya, A., Ali, A. M., \& Kara, A. (2019). Variational mode decomposition-based radio frequency fingerprinting of bluetooth devices. IEEE Access, 7, 144054-144058.

14. Gupta, R., Majhi, S., \& Dobre, O. A. (2018). Design and implementation of a tree-based blind modulation classification algorithm for multiple-antenna systems. IEEE Transactions on Instrumentation and Measurement, 68(8), 3020-3031.

15. Zhang, Z., Hua, Z., \& Liu, Y. (2017). Modulation classification in multipath fading channels using sixth-order cumulants and stacked convolutional auto-encoders. IET communications, 11(6), 910-915.

16. Lee, J. H., Kim, J., Kim, B., Yoon, D., \& Choi, J. W. (2017). Robust automatic modulation classification technique for fading channels via deep neural network. Entropy, 19(9), 454.

17. Gençol, K., Kara, A., \& At, N. (2017). Improvements on deinterleaving of radar pulses in dynamically varying signal environments. Digital Signal Processing, 69, 86-93.

18. Gencol, K., At, N., \& Kara, A. (2016). A wavelet-based feature set for recognizing pulse repetition interval modulation patterns. Turkish Journal of Electrical Engineering \& Computer Sciences, 24(4), 3078-3090.

19. Chang, D. C., \& Shih, P. K. (2015). Cumulants-based modulation classification technique in multipath fading channels. Iet Communications, 9(6), 828-835.

20. Marey, M., \& Dobre, O. A. (2014). Blind modulation classification algorithm for single and multiple-antenna systems over frequency-selective channels. IEEE signal processing letters, 21(9), 1098-1102.

21. Ebrahimzadeh, A., \& Ghazalian, R. (2011). Blind digital modulation classification in software radio using the optimized classifier and feature subset selection. Engineering Applications of Artificial Intelligence, 24(1), 50-59.

22. Yalcinkaya, B. (2020) Performance Analysis of Hierarchical Classification of Modulation Types (Master's thesis, Atilim University) doi: 10.13140/RG.2.2.15329.02400

23. O'Shea, T. J., Roy, T., \& Clancy, T. C. (2018). Over-the-air deep learning based radio signal classification. IEEE Journal of Selected Topics in Signal Processing, 12(1), 168-179.

24. O'shea, T., \& Hoydis, J. (2017). An introduction to deep learning for the physical layer. IEEE Transactions on Cognitive Communications and Networking, 3(4), 563-575.

25. Deepsig. RF DATASETS FOR MACHINE LEARNING [Online]. Available: https://www.deepsig.io/datasets

26. Klein, R. W., Temple, M. A., \& Mendenhall, M. J. (2009). Application of wavelet-based RF fingerprinting to enhance wireless network security. Journal of Communications and Networks, 11(6), 544-555.

27. Geisinger, N. P. (2010). Classification of digital modulation schemes using linear and nonlinear classifiers. NAVAL POSTGRADUATE SCHOOL MONTEREY CA.

28. Zhou, X., Wu, Y., \& Yang, B. (2010). Signal Classification Method Based on Support Vector Machine and High-Order Cumulants. Wirel. Sens. Netw., 2(1), 48-52.

29. Kim, N., Kehtarnavaz, N., Yeary, M. B., \& Thornton, S. (2003). DSP-based hierarchical neural network modulation signal classification. IEEE Transactions on Neural Networks, 14(5), 1065-1071.

30. Zhu, Z., \& Nandi, A. K. (2014). Blind digital modulation classification using minimum distance centroid estimator and non-parametric likelihood function. IEEE Transactions on Wireless Communications, 13(8), 4483-4494.

31. Aslam, M. W., Zhu, Z., \& Nandi, A. K. (2012). Automatic modulation classification using combination of genetic programming and KNN. IEEE Transactions on wireless communications, 11(8), 2742-2750. 
32. Wong, M. D., Ting, S. K., \& Nandi, A. K. (2008, December). Naive bayes classification of adaptive broadband wireless modulation schemes with higher order cumulants. In 2008 2nd International Conference on Signal Processing and Communication Systems (pp. 1-5). IEEE

33. Hazza Alharbi, S. M., Alshebeili, S., \& Alturki, F. (2013). Automatic modulation classification of digital modulations in presence of HF noise.

34. O'Shea, T. J., Corgan, J., \& Clancy, T. C. (2016, September). Convolutional radio modulation recognition networks. In International conference on engineering applications of neural networks (pp. 213-226). Springer, Cham.

35. Wu, Z., Zhou, S., Yin, Z., Ma, B., \& Yang, Z. (2017). Robust automatic modulation classification under varying noise conditions. IEEE Access, 5, 19733-19741.

36. Subbarao, M. V., \& Samundiswary, P. (2020). Performance analysis of modulation recognition in multipath fading channels using pattern recognition classifiers. Wireless Personal Communications, 115(1), 129-151.

37. Baris, B., Cek, M. E., \& Kuntalp, D. G. (2021). Modulation Classification of MFSK Modulated Signals Using Spectral Centroid. Wireless Personal Communications, 1-13. 